Document downloaded from:

http://hdl.handle.net/10251/82084

This paper must be cited as:

Michael, L.; Gómez Barquero, D. (2016). Bit-Interleaved Coded Modulation (BICM) for ATSC 3.0. IEEE Transactions on Broadcasting. 62(1):181-188. doi:10.1109/TBC.2015.2505414.

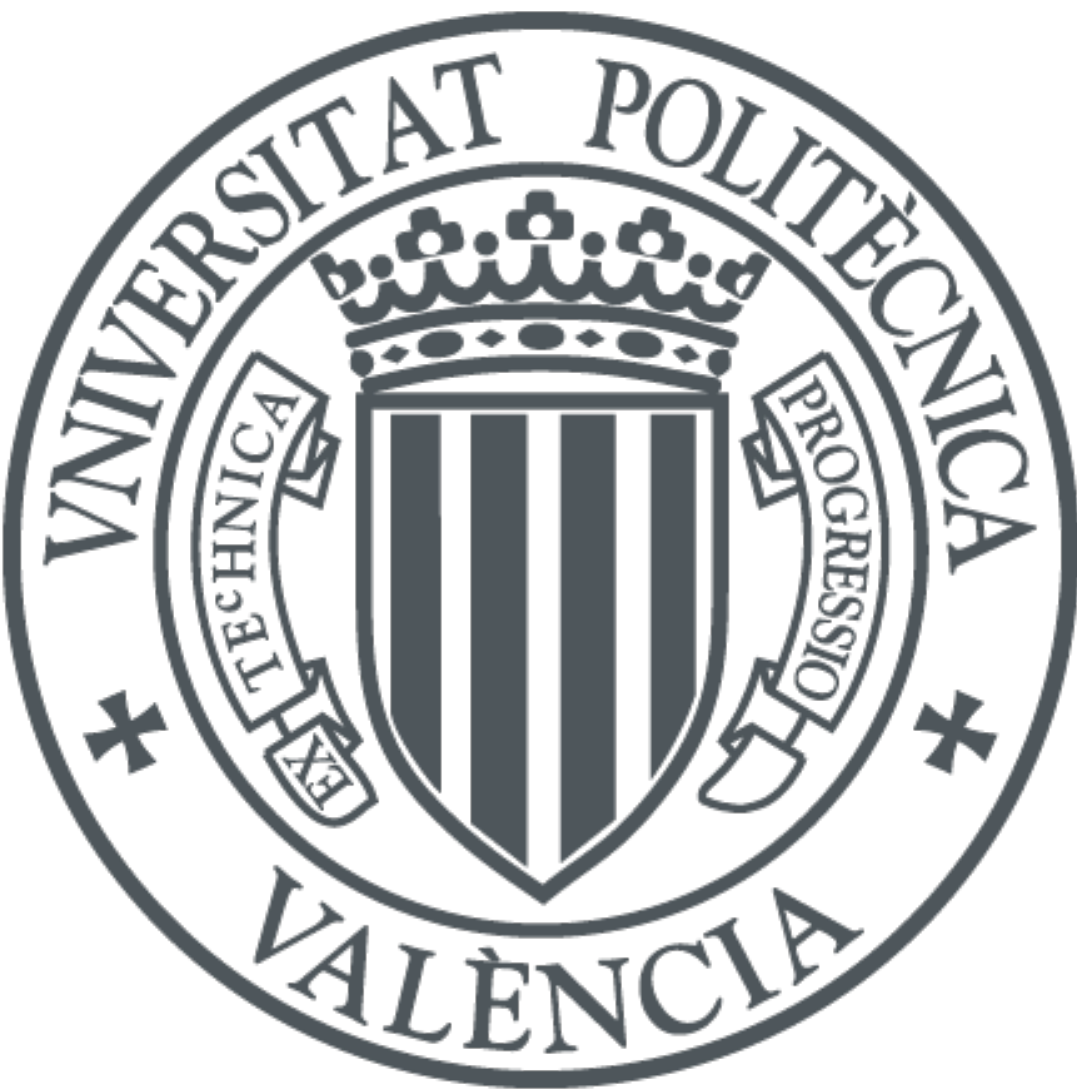

The final publication is available at

http://dx.doi.org/10.1109/TBC.2015.2505414

Copyright Institute of Electrical and Electronics Engineers (IEEE)

Additional Information

"(c) 2016 IEEE. Personal use of this material is permitted. Permission from IEEE must be obtained for all other users, including reprinting/ republishing this material for advertising or promotional purposes, creating new collective works for resale or redistribution to servers or lists, or reuse of any copyrighted components of this work in other works.") 


\title{
Bit-Interleaved Coded Modulation (BICM) for ATSC 3.0
}

\author{
Lachlan Michael and David Gómez-Barquero
}

\begin{abstract}
In this paper we summarize and expound upon the choices made for the BICM (Bit-Interleaved Coded Modulation) part of the next-generation terrestrial broadcast standard known as ATSC 3.0. The structure of the ATSC 3.0 BICM consists of a forward error correcting (FEC) code, bit interleaver and constellation mapper. In order to achieve high efficiency over a wide range of reception conditions and Carrier-to-Noise (C/N) ratio values, several notable new elements have been standardized. First, twenty four original Low-Density Parity Check (LDPC) codes have been designed, with coding rates from 2/15 (0.13) up to 13/15 (0.87) for two code sizes: 16200 bits and 64800 bits. Two different LDPC structures have been adopted; one structure more suited to medium and high coding rates and another structure suited to very low coding rates. Second, in addition to QPSK, Non-Uniform Constellations (NUCs) have been chosen for constellation sizes from 16QAM to 4096QAM to bridge the gap to the Shannon theoretical limit. Two different types of NUCs have been proposed: one-dimensional NUCs (1D-NUC) for 1024- and 4096-point constellations, and two-dimensional NUCs (2D-NUCs) for 16-, 64- and 256-point constellations. 2D-NUCs achieve a better performance than 1D-NUCs but with a higher complexity since they cannot be separated into two independent I/Q components. NUCs have been optimized for each coding rate for the $\mathbf{6 4 8 0 0}$ bits LPDCs. The same constellations are used for 16200 bits LDPCs, although they have been limited up to 256QAM. Finally, a bit interleaver, optimized for each $\mathrm{NUC/coding} \mathrm{rate} \mathrm{combination,} \mathrm{has} \mathrm{been} \mathrm{designed} \mathrm{to} \mathrm{maximize} \mathrm{the}$ performance. The result is a BICM that provides the largest operating range (more than $30 \mathrm{~dB}$, with the most robust mode operating below $-5 \mathrm{~dB} \mathrm{C} / \mathrm{N}$ ) and the highest spectral efficiency compared to any digital terrestrial broadcast system today, outperforming the current state-of-the-art DVB-T2 standard BICM by as much as $1 \mathrm{~dB}$ in some cases. ATSC 3.0 will also provide a considerable increase in the maximum transmission capacity when using the high-order non-uniform constellations such as 1024QAM and 4096QAM, which will represent a major milestone for terrestrial broadcasting since the highest order constellation currently available is uniform 256QAM. This paper describes the coding, modulation and bit interleaving modules of the BICM block of ATSC 3.0, and compares its performance with other DTT standards such as ATSC A/53 and DVB-T2.
\end{abstract}

Index Terms-ATSC 3.0, BICM, Coding, LDPC, Modulation, Non-Uniform Constellations.

Manuscript received July 31, 2015; reviewed September 13, 2015. Parts of this paper have been published in the Proc. IEEE BMSB 2015, Ghent, Belgium.

L. Michael is with the Digital Analog System Development Department, Research \& Development Division, Device Solutions Business Group, Sony Corporation, Atsugi-shi 243-0021 Japan. (e-mail: lachlan.michael@jp.sony.com).

D. Gómez-Barquero is with the Institute of Telecommunications and Multimedia Applications (iTEAM), Universitat Politecnica de Valencia, Valencia 46022, Spain (e-mail: dagobar@iteam.upv.es).

\section{INTRODUCTION}

$\mathrm{B}$ IT-Interleaved Coded Modulation (BICM) is the state-of-the-art pragmatic approach for combining channel coding with digital modulations in fading transmission channels [1], where the modulation constellation can be chosen independently of the coding rate. The structure of the BICM block consists of the serial concatenation of a forward error correction (FEC) code, a bit interleaver, and a constellation mapper. Within the physical layer, the modulation and coding constitute the main portions of the system that affect the overall spectral efficiency, which is a key performance indicator to make an efficient use of the scarce radio spectrum.

The spectral efficiency of the first-generation Digital Terrestrial Television (DTT) standard ATSC (Advanced Television Systems Committee) A/53 [2], currently used in the U.S., Canada, Mexico and South Korea, is far from the theoretical capacity Shannon limit. ATSC A/53 employs 8-level Vestigial Side Band (8VSB) modulation, and a FEC coding scheme based on the concatenation of a convolutional inner code and an outer Reed-Solomon (RS) code. Receiver operation point is approximately $15.0 \mathrm{~dB}$ Carrier-to-Noise $(\mathrm{C} / \mathrm{N})$ ratio to ensure a good reception with Additive White Gaussian Noise (AWGN), and its transmission capacity is about 19.4 Mbps in a $6 \mathrm{MHz}$ radio frequency (RF) channel, which results in a spectral efficiency of $3.23 \mathrm{bps} / \mathrm{Hz}$. Taking into account these values, and using the Shannon's capacity formula:

$$
\text { Capacity }=B W \cdot \log _{2}(1+\Gamma)
$$

where $B W$ is the system bandwidth and $\Gamma$ is the $\mathrm{C} / \mathrm{N}$ in linear units. It can be shown that the operating point of ATSC A/53 is $6.77 \mathrm{~dB}$ and 10.7 Mbps away from the Shannon limit in a 6 $\mathrm{MHz}$ RF channel.

Compared to the first-generation ATSC A/53 DTT standard, the next-generation DTT ATSC standard, known as "ATSC 3.0 " [3], is required to provide at least $30 \%$ capacity increase at the same operating point, and to be significantly more robust [4], in to cope with the broadcast spectrum shortage due to the rapidly growing demand for wireless broadband services and the upcoming broadcast TV spectrum incentive auction in the U.S. [5]. But ATSC 3.0 aims to become the reference terrestrial broadcasting technology worldwide, outperforming existing terrestrial broadcast standards [6], [7], and leveraging recent research into digital terrestrial broadcasting [8]-[10].

Current state-of-the-art terrestrial broadcasting standards are 


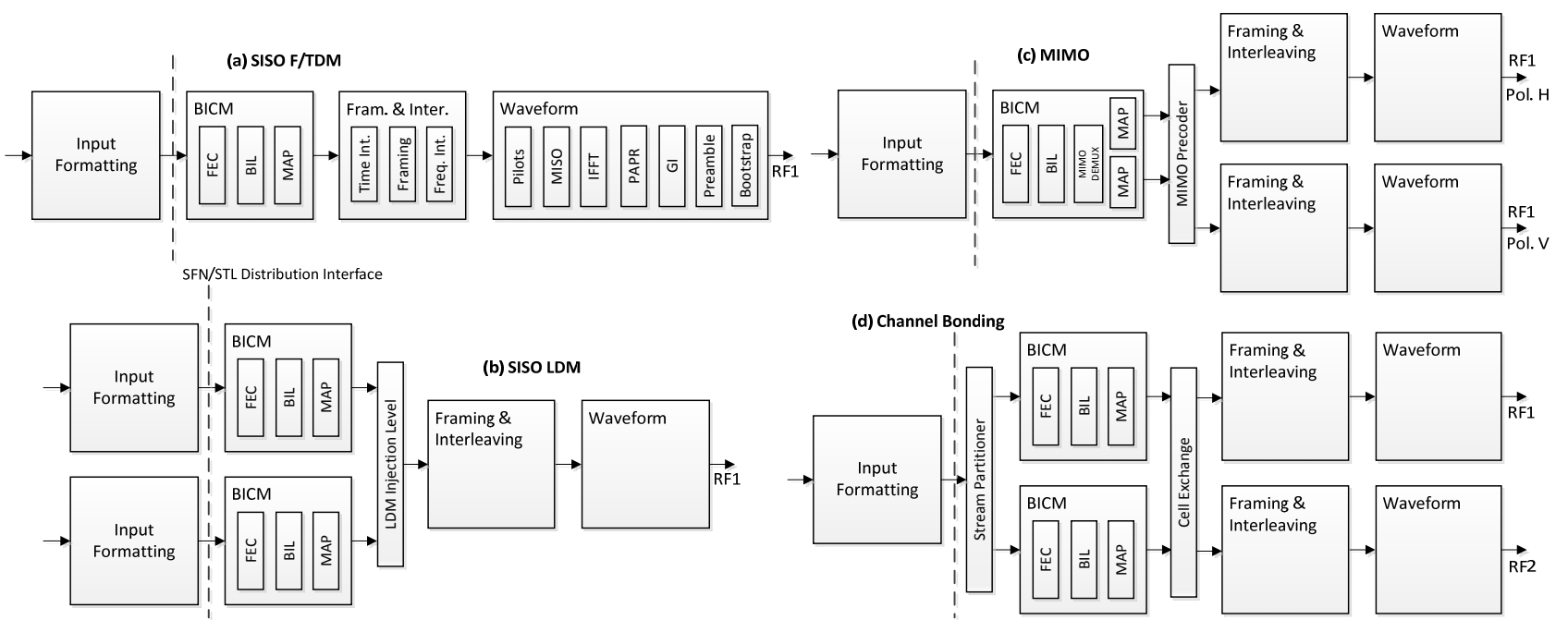

Fig. 1. Block diagrams of ATSC 3.0 physical layer architecture for a single RF channel for SISO F/TDM (a), SISO LDM (b), MIMO (c), and for two RF channel with channel bonding (d). The use of MIMO is not available with LDM and channel bonding.

very close to the Shannon theoretical limit using Low-Density Parity Check (LDPC) codes and implementing uniform QAM (Quadrature Amplitude Modulation) constellations up to 256QAM. However, for ATSC 3.0 several notable new elements have been standardized for the BICM block in order to bridge the remaining gap to the theoretical limit without the need for additional transmission power or bandwidth, and achieve extremely high efficiency over a wide range of reception conditions and $\mathrm{C} / \mathrm{N}$ values. In particular, ATSC 3.0 has adopted:

- New LDPC codes with coding rates from 2/15 (0.13) up to 13/15 (0.87) for two code sizes: 16200 and 64800 bits [11].

- Non-Uniform Constellations (NUCs) from 16-QAM to 4096-QAM, in addition to QPSK. Two different types of NUCs have been adopted: two-dimensional NUCs (2D-NUCs) for 16-, 64- and 256-point constellations, and one-dimensional NUCs (1D-NUC) for 1024- and 4096-point constellations, respectively [12].

- Bit Interleavers (BIL), optimized for each combination of coding rate and constellation pattern.

The result is that the BICM block of ATSC 3.0 provides not only the highest spectral efficiency compared to any DTT system today, but it also provides the largest operating range (more than $30 \mathrm{~dB} \mathrm{C} / \mathrm{N}$ ), and a significant increase in the maximum transmission robustness and transmission capacity, with the most robust mode (QPSK 2/15) operating below $-5 \mathrm{~dB}$ $\mathrm{C} / \mathrm{N}$, and the highest capacity mode (4096QAM 1D-NUC $13 / 15$ ) with a spectral efficiency of $10.4 \mathrm{bps} / \mathrm{Hz}$. As a reference, the most robust and highest capacity modes of DVB-T2 are QPSK 1/2 and uniform 256QAM 5/6, respectively. Compared to ATSC A/53, ATSC 3.0 is almost $4 \mathrm{~dB}$ and $7 \mathrm{Mbps}$ closer to the Shannon limit in a $6 \mathrm{MHz}$ RF channel, and the gain compared to DVB-T2 reaches up to $1 \mathrm{~dB}$ in some cases.

This paper describes the coding, modulation and bit interleaving blocks of the BICM module of ATSC 3.0, and compares its performance with the ATSC A/53 and DVB-T2 standards. A summary version of the paper was presented in [13]. The rest of the paper is structured as follows. Section II presents a brief overview of the ATSC 3.0 physical layer. Section III describes the BICM methodology followed within the standardization process. Section IV, Section V and Section VI present the FEC coding, modulation and bit interleaving, respectively. Section VII presents illustrative results of the BICM performance of ATSC 3.0, and compares it with ATSC A/53 and DVB-T2. Finally, Section VIII concludes the paper.

\section{ATSC 3.0 PHYSICAL LAYER OVERVIEW}

Fig. 1 shows the different block diagrams of the ATSC 3.0 physical layer architecture [3]. As can be seen in the figure, ATSC 3.0 allows for the optional use of superposition modulation LDM (Layer-Division Multiplexing) [15] in addition to FDM (Frequency-Division Multiplexing) and TDM (Time-Division Multiplexing) for SISO (Single-Input Single-Output), MIMO (Multiple-Input Multiple-Output) technology with cross/dual-polarized transmission [16], and channel bonding [17], which consists in combining two RF channels.

The BICM module is one of the most important modules as it provides the error correction capability for the system, allowing the broadcaster multiple choices to trade off robustness for capacity. The improvements in this block are the major reasons for improvements in efficiency compared to both the previous generation ATSC standard A/53 and improvements compared to DVB-T2. An outline of the system architecture follows.

The input formatting module takes input IP and other data packet types and forms it into physical layer containers, known as baseband frames. The SFN (Single Frequency Network) STL (Studio-Transmitter Link) distribution interface guarantees that the output of the physical layer is deterministic for a given input, enabling SFNs. The framing and interleaving module performs the time and frequency interleaving and constructs the physical layer frame. The waveform module consists of the pilot insertion, FFT (Fast Fourier Transform) and guard interval, preamble, and allows for the optional use of Multiple-Input Single Output (MISO) [14] and peak-to-average power ratio (PAPR) reduction techniques. 


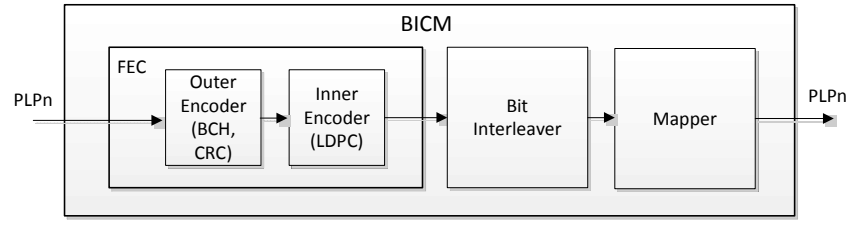

Fig. 2. Block diagram of the ATSC 3.0 BICM module for SISO.

Fig. 2 depicts the block diagram of the BICM module for a single Physical Layer Pipe (PLP) for SISO, which, as noted previously, consists of the FEC, bit interleaver and constellation mapping. For MIMO there is also an antenna stream demultiplexer, which distributes the output bits from the bit interleaver into two constellation mappers, one for each transmit antenna, see Fig. 1(c). The input to the BICM module is a sequence of baseband frames carrying randomized data. The FEC block is divided into an outer encoder and an inner encoder. The inner code is an LDPC code and its use is mandatory. For the outer code, there are several choices possible. First, the use of $\mathrm{BCH}$ (Bose-ChaudhuriHocquengham) code; second the use of a 32 bit CRC (Cyclic Redundancy Check) and third not using any outer coding.

Regarding the support of multiple PLPs in ATSC 3.0, on the transmitter side a maximum of 64 PLPs can be simultaneously transmitted in a single radio frequency channel, however, a single service consists of up to 4 PLPs. This means that receivers must be able to receive and decode at least a minimum of 4 PLPs (i.e., one service). PLPs of the same service share a common time interleaver memory of $2^{19}$ cells (constellation symbols). In ATSC 3.0 an additional feature has been added, similar to NGH, that allows for doubling of the time interleaver depth, we call it "extended interleaving" here. In extended interleaving in ATSC 3.0, double the number of cells is allowed, up to $2^{20}$ cells for single PLP for QPSK only. The feature of extended interleaving is that the actual physical memory need not be doubled, but by reducing the bits assigned to each cell, two QPSK cells can fit into the same physical memory as one cell for the higher modulations. This achieves higher time interleaver depth without increasing physical memory.

\section{BICM STANDARDIZATION METHODOLOGY}

For each BICM technology item, a work plan was established and over the course of approximately one year the technology making up the BICM part was chosen by consensus from the different proposals. A piecewise methodology was followed to ensure the most suitable technology was chosen for each part of the specification, starting with the FEC codes, following with the constellations and finishing with the bit interleavers. The main indicator in the selection of technologies was performance, leaving complexity and other factors as a secondary consideration. This made the task of technology choice to be primarily a matter of determining the performance of each technology proposal compared to other proposals. The performance was confirmed not only by the proponent but by multiple participants. To this end, the first step in the choice of technology was to confirm full disclosure about each part, such that each participant could freely implement other proposals and perform cross-check simulations. This approach ensured transparency and gave a high degree of confidence in the final results.

Regarding the initial selection of the FEC codes, first the constellation was fixed at QPSK and no bit interleavers were used. Other evaluation conditions included setting the number of decoder iterations to 50, and the target for bit error rate (BER) and frame error rate (FER) curves down to $10^{-8}$ and $10^{-6}$, respectively were decided by consensus. Two channel models were used in the comparisons: AWGN and i.i.d. Rayleigh, to ensure that the chosen codes would be suitable for a wide range of applications from static to portable reception.

All 136 FEC code proposals were then compared together in a monolithic manner, classifying the FEC codes in two groups consisting of shorter code lengths (less than 20,000 bits) and longer code lengths (larger than 50,000 bits). For each group, similar code rates were grouped together and compared. From the best performing code in each code rate group, those codes within $0.1 \mathrm{~dB}$ were allowed to remain for a second round of comparisons, which included detailed cross-checks and analysis of other factors including complexity. Finally, 24 LDPC codes were chosen, with coding rates from 2/15 (0.13) up to $13 / 15$ (0.87) for two code sizes of 16200 and 64800 bits. Two different code structures were chosen to cover both the medium/high and low code rates.

The next step consisted of choosing the constellations. Both uniform and non-uniform QAM constellations were proposed, although it became clear during the examination that some NUCs were clearly superior in performance [18], [19]. In order to limit the complexity at the receiver side, two-dimensional non-uniform constellations (2D-NUCs) were adopted for 16-, 64- and 256-point constellations, and one-dimensional non-uniform constellations (1D-NUCs) for 1024- and 4096-point constellations. QPSK was adopted as the most robust constellation. A different NUC was designed for each coding rate for LDPCs of 64800 bit length. The same constellations were adopted for 16200 bit length LDPCs, although the constellation size was limited up to 256QAM, since the main use case proposed for the shorter length codes was reduced implementation complexity.

The constellations were jointly evaluated with the bit interleavers using the previously agreed FEC codes. Each constellation and bit interleaver is potentially different for each coding rate, although a common bit interleaver structure was agreed before the evaluations to reduce unneeded implementation complexity.

The final step consisted of examining the 72 MODCOD (modulation and coding) combinations for long codes and the 48 MODCOD combinations for short codes, in order to reduce the number of combinations to a manageable set without losing flexibility. MODCODs were compared in terms of AWGN and Rayleigh robustness (C/N threshold) and spectral efficiency, and finally 46 MODCODs for long codes and 29 MODCODs for short codes were chosen to be required combinations that must be mandatorily implemented by all transmitters and receivers. Nevertheless, to allow for complete flexibility for unforeseen future situations, all the MODCOD combinations 


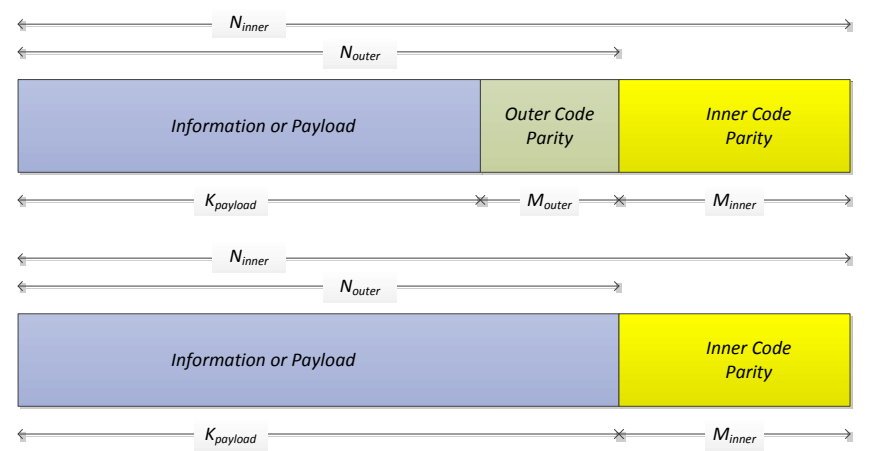

Fig. 3. Format of ATSC 3.0 FEC frame when BCH or CRC is used (top) and format when only the inner LPDC code is used (bottom).

will remain in the standard as options.

\section{ATSC 3.0 CODING}

The FEC of the BICM is formed by concatenation of an outer code and an inner code with the information part. The outer code is either a $\mathrm{BCH}$ code, a CRC or none, whereas the inner code is a LDPC code. BCH, CRC and LDPC codes are systematic codes, such that the information part is contained within the codeword. The resulting codeword is thus a concatenation of information or payload part, BCH or CRC parities and LDPC parities. Fig. 3 shows the structure of the FEC frames at the output of the FEC sub-block when BCH or CRC are used and when no outer code is used.

For maximum flexibility and to achieve higher throughput when the error correction capability of the inner code is deemed sufficient, the outer code can be either a $\mathrm{BCH}$ with the ability to correct up to 12 bit errors in a LDPC codewords, a CRC check, or none at all. Nevertheless, the use of the $\mathrm{BCH}$ is expected to be the most common use case, as it provides additional error correction as well as error detection. CRC is provided to give the choice of improved efficiency, however no additional error correction is available, only error detection. Table I outlines examples of the efficiency gain that can be achieved using the CRC or without any outer code compared to the expected reference case using $\mathrm{BCH}$.

Regarding the inner LDPC, two different structures have been chosen [11]. One structure is similar to that used in DVB-T2, which provides excellent performance at medium and high code rates, and another slightly different LDPC structure which showed excellent performance at very low code rates (in general, less than or equal to 5/15). The use of two different inner code structures in the same standard is quite unusual, but the wide range of code rates chosen, from $3 / 15$ up to $13 / 15$, justifies this approach. Two different lengths of LDPC code have been defined: 64800 and 16200 bits, as in DVB-T2 [6], [8]. In general, 64800 bit codes are expected to be employed as the performance is better, although for applications where latency is critical, or a simpler encoder and decoder structure is preferred, 16200 bit codes may also be used. The 16200 bit codes adopted in ATSC 3.0 have lower latency but worse performance than the 64800 bit codes.
TABLE I

EFFICIENCY GAIN OF CRC AND NO OUTER CODE COMPARED TO BCH

\begin{tabular}{c|c|c|c}
\hline \hline $\begin{array}{c}\text { LDPC } \\
\text { Codeword }\end{array}$ & $\begin{array}{c}\text { LDPC } \\
\text { Code Rate }\end{array}$ & $\begin{array}{c}\text { Outer } \\
\text { CRC }\end{array}$ & $\begin{array}{c}\text { No Outer } \\
\text { Code }\end{array}$ \\
\hline & $13 / 15$ & $0.29 \%$ & $0.34 \%$ \\
64800 & $10 / 15$ & $0.37 \%$ & $0.45 \%$ \\
bits & $7 / 15$ & $0.53 \%$ & $0.64 \%$ \\
& $2 / 15$ & $1.86 \%$ & $2.27 \%$ \\
\hline & $13 / 15$ & $0.97 \%$ & $1.21 \%$ \\
16200 & $10 / 15$ & $1.26 \%$ & $1.58 \%$ \\
bits & $7 / 15$ & $1.81 \%$ & $2.27 \%$ \\
& $2 / 15$ & $6.39 \%$ & $8.43 \%$ \\
\hline \hline
\end{tabular}

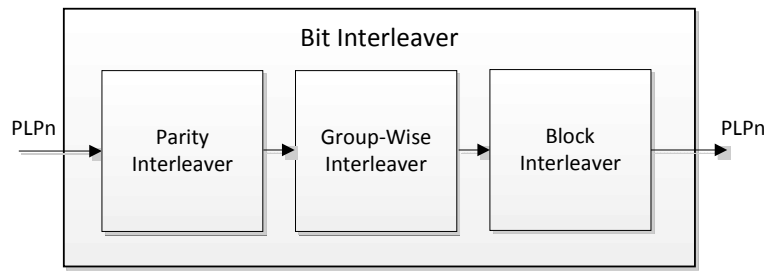

Fig. 4. ATSC 3.0 three stage bit interleaver block diagram.

\section{ATSC 3.0 BIT INTERLEAVING}

The role of the bit interleaver in the BICM chain is to match the output of the LDPC codewords to the constellations. The bit interleaver affects the performance and also the HW implementation. ATSC 3.0 has adopted a 3-stage bit interleaver structure introduced in the MIMO profile of DVB-NGH [7], [9]. Fig. 4 shows the block diagram of the bit interleaver, which consists of a parity interleaver followed by a group-wise interleaver followed by a block interleaver. This structure allows for parallel LDPC decoding while optimizing the performance of the FEC codes to any constellation.

The role of the parity interleaver is to convert the staircase structure of the parity-part of the LDPC parity-check matrix into a quasi-cyclic structure similar to the information-part of the matrix enabling parallel decoding. The group-wise interleaving allows optimizing the combination between the FEC code and the constellation, and hence it is optimized for each combination of modulation and LDPC coding rate. Finally the block interleaver provides the final allocation from bits to constellation symbols.

\section{ATSC 3.0 MODULATION}

ATSC 3.0 has adopted complex-valued quadrature amplitude modulation (QAM) constellations. For the highest robustness level, quaternary phase shift keying (QPSK) is used. For higher order constellations with a higher spectral efficiency but a lower robustness level, non-uniform constellations from 16QAM up to 4096QAM are defined with customized constellations for each LDPC code rate [12].

Uniform QAM constellations, characterized with uniform spacing between constellations points and square shape of the constellations, have been traditionally used in many communication standards because of their simplicity for encoding and decoding. However, there is a significant gap between the BICM capacity of uniform QAM constellations 


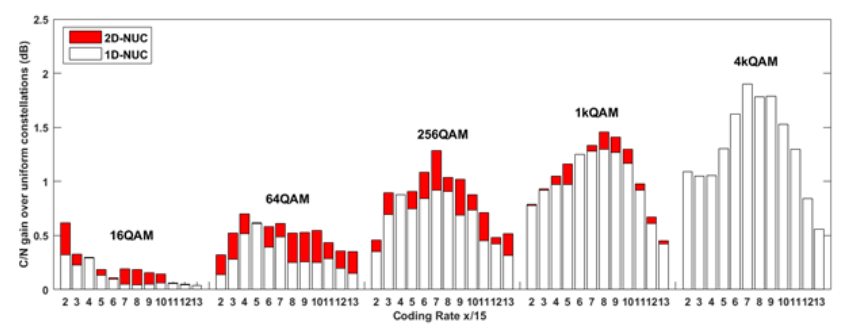

Fig. 5. Waterfall $\mathrm{C} / \mathrm{N}$ gain of $1 \mathrm{D}$ and $2 \mathrm{D}$ non-uniform constellations over uniform constellations for 16QAM, 64QAM, 256QAM, 1024QAM (1kQAM) and 4096QAM (4kQAM) in an AWGN channel. 4kQAM results shown are only for $1 \mathrm{D}$.

and the theoretical Shannon limit, which increases with the modulation order. NUCs can be used to reduce this gap and provide a better performance, reducing the required SNR with respect to the corresponding uniform constellation (i.e., providing a coverage gain). Consequently, the gain provided by NUCs increases with the constellation size.

For 1D-NUCs, 1-dimensional refers to the fact that a 2-dimensional QAM constellation can be separated into two 1-dimensional PAM (Pulse-Amplitude Modulation) constellations, one for each in-phase (I) and quadrature (Q) component, which can be demapped separately. Hence, two PAM demappers are sufficient to demap 1D-NUCs. These constellations can be solely described by the real axis level of each point (the constellation points are deduced by symmetry), and they are designed by relaxing the spacing constraint of the uniform constellation.

2D-NUCs are designed by relaxing also the square shape constraint of uniform constellations, achieving a better performance than 1D-NUCs but with a higher receiver complexity since they cannot be separated into two independent I/Q components. Hence, a full 2D demapper is needed to demap 2D-NUCs. Nevertheless, it should be pointed out that DVB-T2 requires also a 2D demapper up to 256QAM due to the use of rotated constellations [20].

Fig. 5 shows an example of the potential $\mathrm{C} / \mathrm{N}$ gain of 1D-NUCs and 2D-NUCs in an AWGN channel. It can be seen that the majority of the gain can be obtained by using 1D-NUCs, especially for 256QAM and 1024QAM, although the extension to 2D-NUCs does provide an additional gain, being most relevant for 16QAM and 64QAM. For the ATSC 3.0 specification, it was agreed to use 2D-NUCs from 16QAM up to 256QAM, and use 1D-NUCs for 1024QAM and 4096QAM, in order to reduce the demapper complexity for these larger constellations.

Each NUC is optimized for a specific code rate for 64800 bits LDPCs, since the optimum shape of the constellation depends on the operating $\mathrm{C} / \mathrm{N}$ [12]. Fig. 6 shows an example of the adopted NUCs, a 2D-NUC for 16QAM and a 1D-NUC for 1024QAM for the LDPC code rate 6/15. 2D-NUCs often resemble circular constellations, since three quadrants are constructed by symmetry from a single quadrant designed to approach a Gaussian distribution to maximize the channel
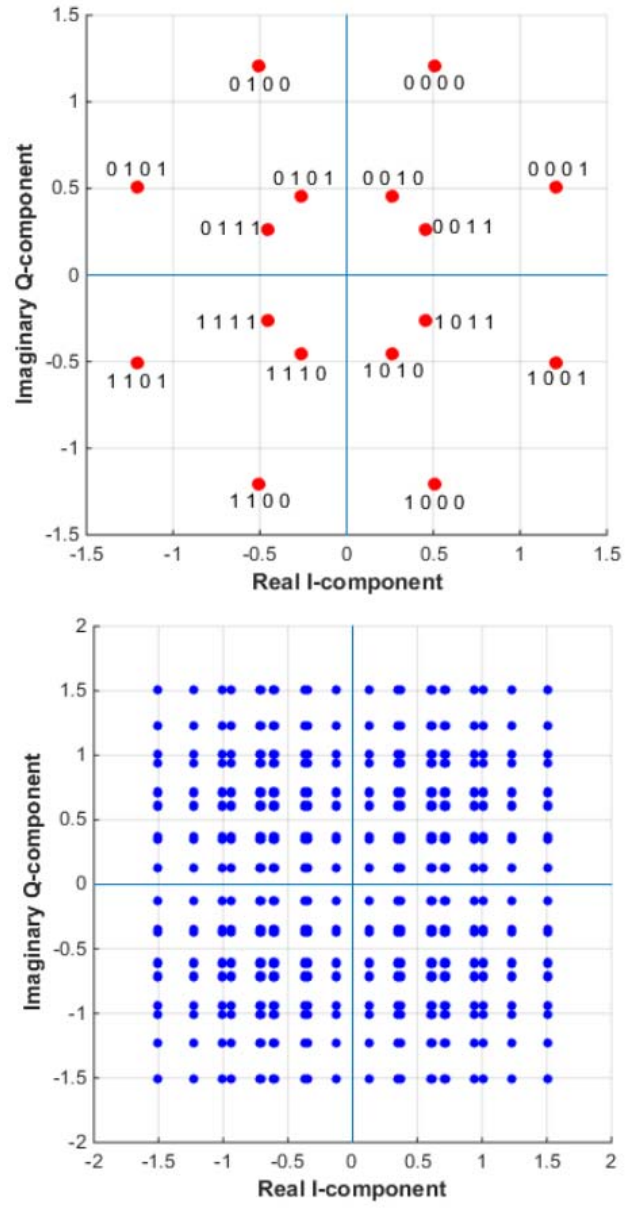

Fig. 6. Example of NUCs for 16QAM (top) and 1024QAM (bottom) for LDPC code rate 6/15.

capacity. It is worth to point out that for very low coding rates (and hence lower $\mathrm{C} / \mathrm{N}$ ), the constellation shape becomes a lower order modulation with less constellation points.

It should be pointed out that ATSC 3.0 has not adopted the use of rotated 2D-NUCs because the gain was limited to the lower constellations and high code rate combinations, which showed overall worse performance compared to using lower code rates and higher constellations.

\section{ATSC 3.0 BICM PERFORMANCE}

Fig. 7 shows the performance in terms of spectral efficiency in bits per second per Hertz (bps/Hz) versus symbol energy per symbol to noise ratio $\left(E_{s} / N_{0}\right)$ of the longer LDPC codes $(64800$ bits) and shorter LDPC codes (16200 bits) without any outer code in AWGN channel. The Shannon capacity limit is also shown as upper bound for reference. In the figures, it can be seen that the BICM for the longer codes performs very close to the Shannon limit. The gap is almost negligible for Es/N0 ratios below $5 \mathrm{~dB}$, whereas for signal-to-noise ratios larger than $5 \mathrm{~dB}$ the gap is about $1 \mathrm{~dB}$. The performance difference between the longer and the shorter LDPC codes in AWGN is about $0.35 \mathrm{~dB}$. 

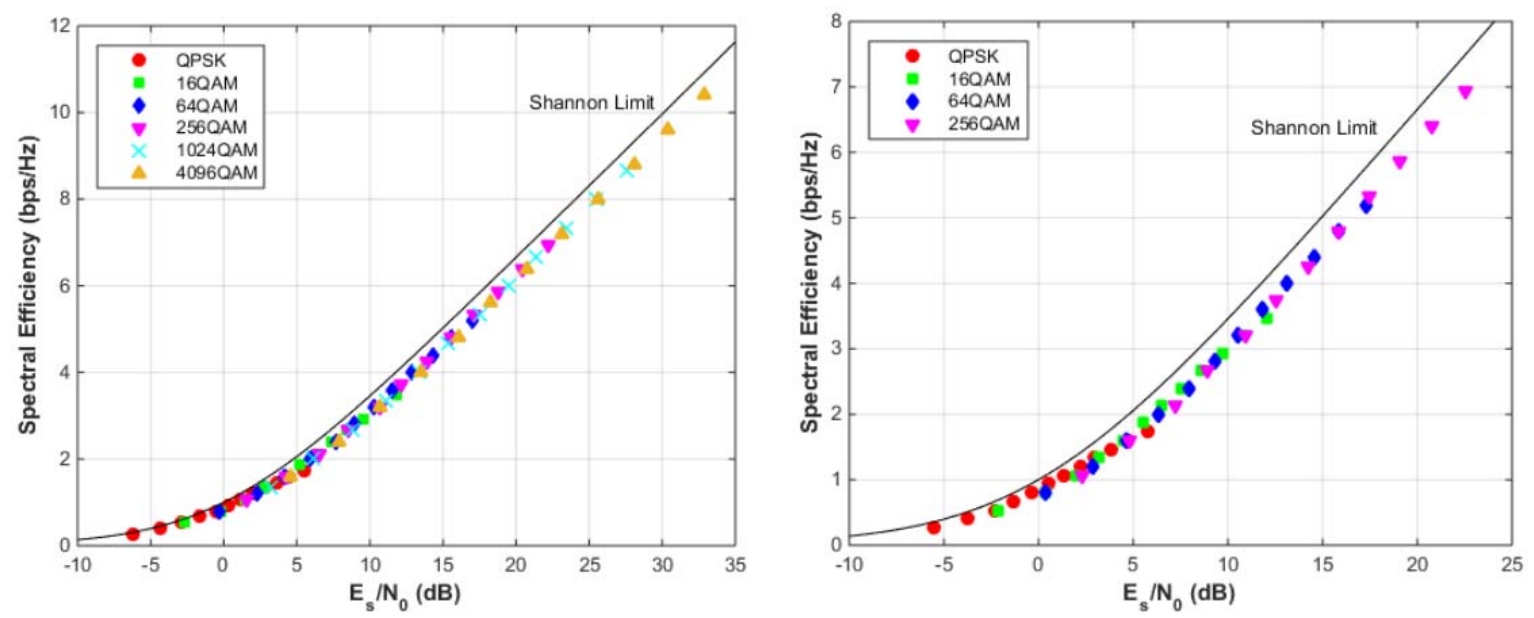

Fig. 7. Capacity plot of ATSC 3.0 BICM performance in AWGN channel. LDPC codeword size 64800 bits (left), 16200 bits (right). No outer code.
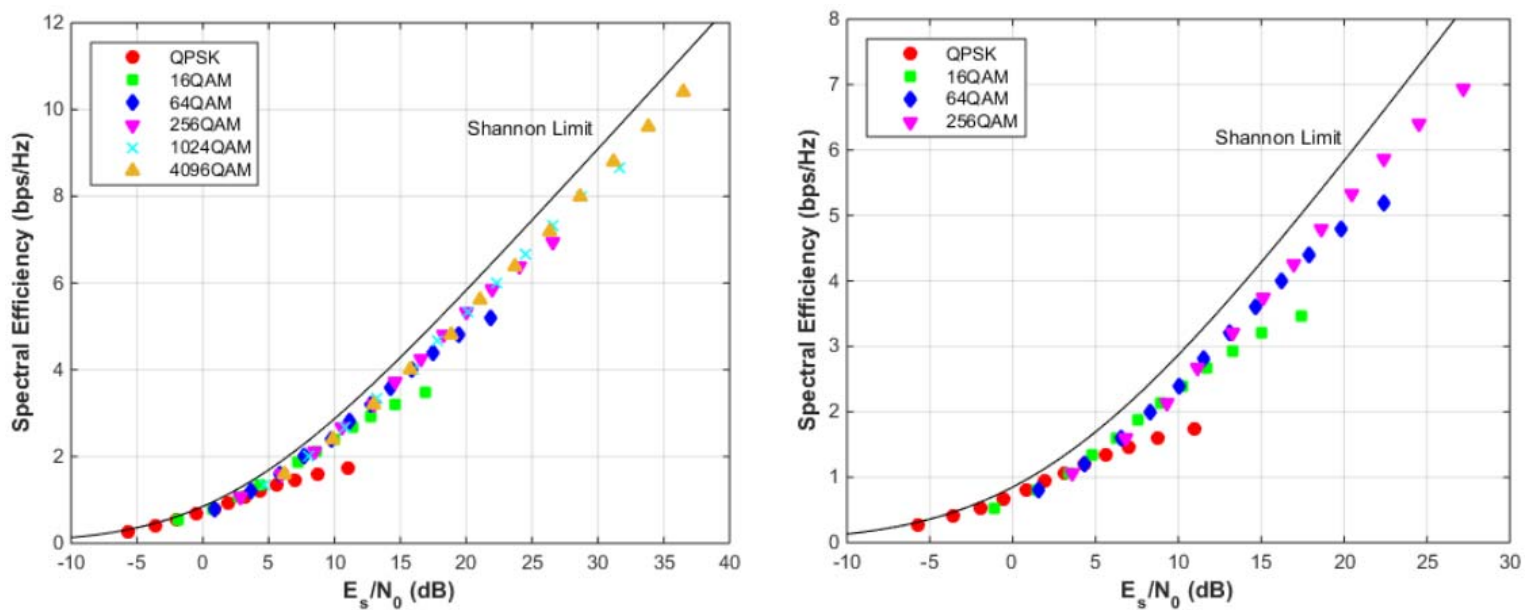

Fig. 8. Capacity plot of ATSC 3.0 BICM performance in Rayleigh channel. LDPC codeword size 64800 bits (left), 16200 bits (right). No outer code.

Fig. 8 shows the capacity plot of the BICM module of ATSC 3.0 for longer and shorter LDPC codes without any outer code in Rayleigh channel. In the figures it can be noted that higher coding rates have a noticeably worse performance, with a larger gap to the Shannon capacity limit. However, the gap of the best MODCODs for longer codes is still around $1 \mathrm{~dB}$ to the upper bound. The performance degradation of the shorter codes compared to the longer codes in Rayleigh channel is about 0.5 $\mathrm{dB}$.

Table II shows the mandatory MODCODs for both the longer and shorter LDPC codes. These were chosen taking into account the performance of each MODCOD in both AWGN and Rayleigh channels. The number of mandatory MODCODs was reduced by almost 65\% for the longer codes (from 72 combinations down to 48), and almost $40 \%$ for the shorter codes (from 48 down to 29).

Fig. 9 compares the capacity in Mbps in a $6 \mathrm{MHz}$ channel provided by the BICM of ATSC 3.0 (for longer LDPCs) with the performance of the current ATSC terrestrial system A/53 [3] and the performance of the current most advanced terrestrial broadcasting standard DVB-T2 [6]. Due to the wide scale of the left graph in Fig. 9, a close-up showing a selection of ATSC 3.0 points for 2D-NUC 256QAM (specifically coding rates from 6/15 to 13/15) compared to DVB-T2 points (coding rates1/2,
TABLE II

MANDATORY MODCODS FOR 64800 BITS $(\boldsymbol{v})$ AND 16200 BITS $(\boldsymbol{*}) \quad$ LDPC

\begin{tabular}{|c|c|c|c|c|c|c|}
\hline \multicolumn{7}{|c|}{ CODES } \\
\hline Code & 4 & 16 & 64 & 256 & 1024 & 4096 \\
\hline Rate & QAM & QAM & QAM & QAM & QAM & QAM \\
\hline $2 / 15$ & $\boldsymbol{V} *$ & & & & & \\
\hline $3 / 15$ & $\boldsymbol{V} *$ & & $\nu$ & & & \\
\hline $4 / 15$ & $\nu *$ & $\checkmark$ & $\checkmark$ & $\checkmark$ & & \\
\hline $5 / 15$ & $V *$ & $v *$ & $\nu x$ & $V *$ & $\checkmark$ & \\
\hline $6 / 15$ & $\nu *$ & $x$ & $\nu x$ & & & \\
\hline $7 / 15$ & $\checkmark x$ & $\checkmark x$ & $\checkmark x$ & $v x$ & $\boldsymbol{\nu}$ & $\nu$ \\
\hline $8 / 15$ & $V *$ & $v x$ & $\nu x$ & $V *$ & $\checkmark$ & \\
\hline $9 / 15$ & $v x$ & $\checkmark$ & $\nu x$ & $v *$ & $\nu$ & $\checkmark$ \\
\hline $10 / 15$ & & & $\nu x$ & $\nu *$ & $\checkmark$ & \\
\hline $11 / 15$ & $\nu$ & $\boldsymbol{V} x$ & $\nu x$ & $\boldsymbol{V} *$ & $\checkmark$ & $\checkmark$ \\
\hline $12 / 15$ & & & & $\nu x$ & $\checkmark$ & $\checkmark$ \\
\hline $13 / 15$ & & & & $V *$ & $\checkmark$ & $\checkmark$ \\
\hline
\end{tabular}

3/5, 2/3, 3/4, 4/5 and 5/6) for uniform 256QAM is also shown.

Compared to ATSC 1.0 (A/53), which has only one operating point, ATSC 3.0 shows advantages in terms of flexibility due to the larger number of operating points. Furthermore, the increase in performance is dramatic, with an increase of more than $5 \mathrm{Mbps}$ in throughput at a slightly lower $\mathrm{E}_{\mathrm{s}} / \mathrm{N}_{0}$ point, or close to $9 \mathrm{Mbps}$ improvement in throughput at a 

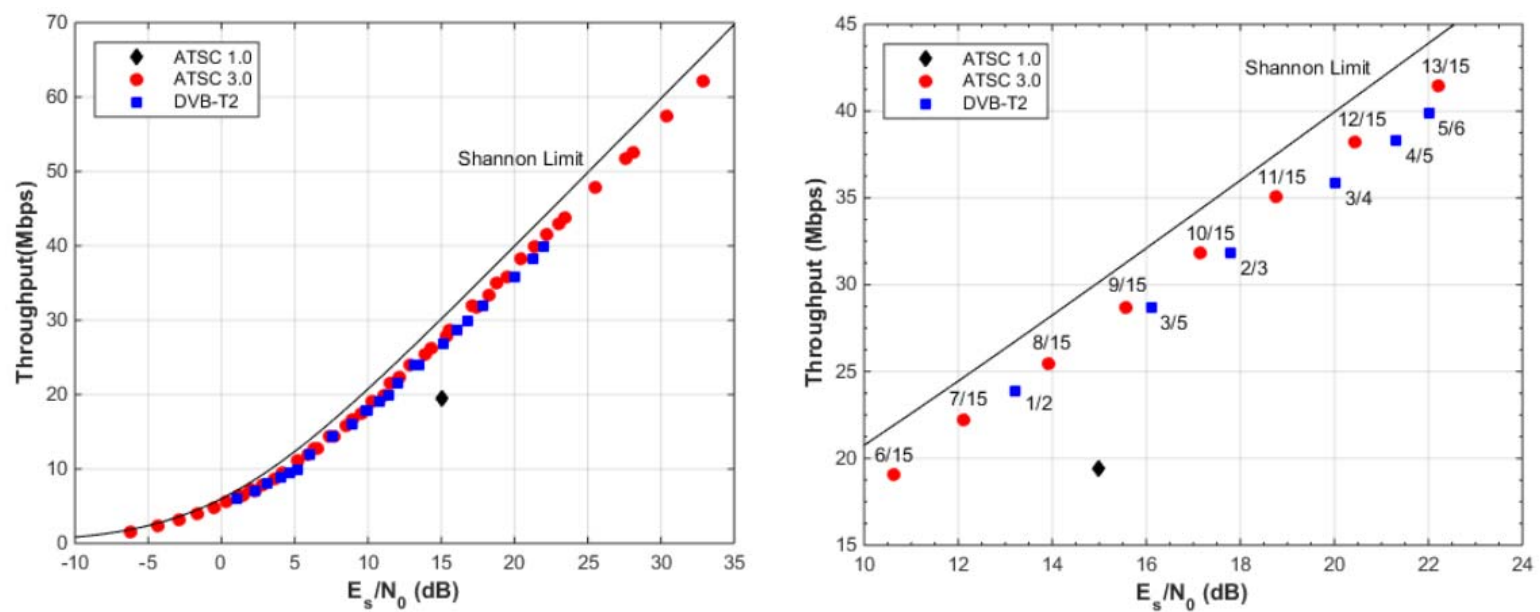

Fig. 9. BICM throughput comparison in a $6 \mathrm{MHz}$ channel between ATSC 3.0, DVB-T2 and ATSC 1.0 in an AWGN channel. LDPC codeword size 64800 bits. Outer code BCH. Figure on the right side is a zoom of the figure on the left side showing only 256QAM points for DVB-T2 and ATSC 3.0.

slightly higher $\mathrm{E}_{\mathrm{s}} / \mathrm{N}_{0}$. It should be pointed out that the comparison with $\mathrm{A} / 53$ is not entirely fair, since OFDM waveform overheads such as guard interval, pilot sub-carriers, and preamble are not considered, but the attractiveness of ATSC 3.0 for broadcasters currently using A/53 is obvious.

Compared to the DVB-T2 standard, two aspects should be noted. Firstly, ATSC 3.0 provides a larger range of operation in terms of robustness and capacity. The most robust MODCODs cover negative SNRs to ensure extremely robust signals, while at the very high SNR range the throughput extends to more than $60 \mathrm{Mbps}$ in a $6 \mathrm{MHz}$ channel that allows exploiting very good reception conditions (e.g., line of sight). Secondly, it can be seen that the performance of the ATSC 3.0 always outperforms that of DVB-T2. Furthermore, it should be stressed that the results for DVB-T2 are taken from [21], which uses so-called "genie-aided" demapping while the results for ATSC 3.0 use the sum-product floating point decoding algorithm. Hence, the results for DVB-T2 may therefore be slightly optimistic for realistic implementations.

To further highlight the potential performance gain of ATSC 3.0 compared to DVB-T2, bit error rate (BER) and frame error rate (FER) physical layer simulations were performed using the same modulation (256QAM) and coding rate (3/5 for DVB-T2 and 9/15 for ATSC 3.0), using the same LDPC sum-product floating point decoding algorithm. Results are shown in Fig. 10. It can be seen that more than $1 \mathrm{~dB}$ gain in an AWGN channel can be realized for exactly the same throughput. This modulation and code rate is representative of the operating point chosen for fixed rooftop reception in most DTT networks worldwide (approximately $16 \mathrm{~dB}$ in an AWGN channel). While the new LDPC codes for the ATSC 3.0 provide some spectral efficiency gain, the majority of the gain comes from the use of non-uniform constellations.

\section{CONCLUSIONS}

The paper has provided an overview of the coding, bit interleaving and modulation of the BICM block of the next-generation U.S. terrestrial broadcasting standard ATSC 3.0. The new technologies adopted in ATSC 3.0 including

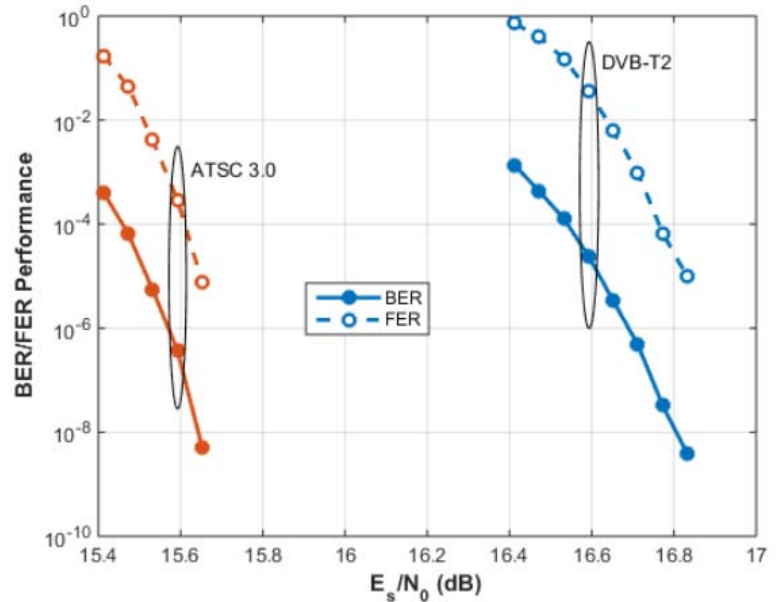

Fig. 10. Comparison of BER/FER performance for ATSC 3.0 and DVB-T2. LPDC codeword size 64800 bits with code rate 9/15, modulation 256QAM.

LDPC codes, bit interleavers and non-uniform constellations, make ATSC 3.0 with superior capacity and coverage performance compared to any existing digital terrestrial broadcasting standard.

In particular, the BICM block of ATSC 3.0 provides a $\mathrm{C} / \mathrm{N}$ operating range of more than $30 \mathrm{~dB}$, with the most robust mode operating below $-5 \mathrm{~dB} C / \mathrm{N}$, a spectral efficiency very close to the theoretical Shannon limit, less than $1 \mathrm{~dB}$ away in both AWGN and Rayleigh channel, and a maximum transmission capacity of 10.4 bits per second per Hertz using one dimensional non-uniform 4096QAM at a coding rate 13/15.

\section{REFERENCES}

[1] A. Guillen i Fabregas, A. Martinez, and G. Caire, "Bit-interleaved coded modulation," Foundations and Trends in Communications and Information Theory, vol. 5, no. 1-2, pp. 1-144, 2008.

[2] ATSC A/53, “ATSC Digital Television Standard,” Parts 1-6, Jan. 2007.

[3] L. Fay, L. Michael, D. Gomez-Barquero, N. Ammar, and W. Caldwell, "An Overview of the ATSC 3.0 Physical Layer Specification," IEEE Transactions on Broadcasting, 2016.

[4] ATSC, Technical Document TG3-S31087r7, "System Requirements for ATSC 3.0,” Nov. 2013. 
[5] D. Gomez-Barquero and M. W. Caldwell, "Broadcast Television Spectrum Incentive Auctions in the U.S.: Trends, Challenges and Opportunities,” IEEE Comm. Mag., vol. 53, no. 7, pp. 50-56, July 2015.

[6] ETSI EN 302755 v1.3.1, "Digital Video Broadcasting (DVB); Frame structure channel coding and modulation for a second generation digital terrestrial television broadcasting system (DVB-T2),” April 2012.

[7] DVB BlueBook A160, "Next Generation broadcasting system to Handheld, physical layer specification (DVB-NGH),” Nov. 2012.

[8] I. Eizmendi, et al., "DVB-T2: The Second Generation of Terrestrial Digital Video Broadcasting System,” IEEE Trans. Broadcast., vol. 60 no. 2, pp. 258-271, Jun. 2014.

[9] D. Gomez-Barquero, C. Douillard, P. Moss, and V. Mignone, "DVB-NGH: The Next Generation of Digital Broadcast Services to Handheld Devices,” IEEE Trans. Broadcast., vol. 60, no. 2, pp. 246-257, Jun. 2014.

[10] D. Gomez-Barquero (Ed.), "Next Generation Mobile Broadcasting," Boca Raton, FL, USA: CRC Press, 2013.

[11] K,-J. Kim, et. al, "Low Density Parity Check Codes (LDPC) for ATSC 3.0,"IEEE Transactions on Broadcasting, 2016.

[12] N. Loghin, et. al, "Non-Uniform Constellations for ATSC 3.0," IEEE Transactions on Broadcasting, 2016.

[13] L. Michael and D. Gomez-Barquero, "Modulation and Coding for ATSC 3.0,” Proc. IEEE BMSB, Ghent, Belgium, 2015.

[14] S. Lopresto, R. Citta, D. Vargas, and D. Gomez-Barquero, "Transmit Diversity Code Filter Sets (TDCFS), a MISO Antenna Frequency Pre-Distortion Scheme for ATSC 3.0,” IEEE Transactions on Broadcasting, vol. 62, no. 1, 2016.

[15] S.-I. Park, et. al, "Low Complexity Layered Division Multiplexing System for ATSC 3.0,” IEEE Transactions on Broadcasting, vol. 62, no. $1,2016$.

[16] D. Gomez-Barquero, et. al, "MIMO for ATSC 3.0, an Overview," IEEE Transactions on Broadcasting, vol. 62, no. 1, 2016.

[17] L. Stadelmeier, D. Schneider, J. Zollner, and J. J. Gimenez, "Channel Bonding for ATSC 3.0,” IEEE Transactions on Broadcasting, vol. 62, no. $1,2016$.

[18] J. Zöllner and N. Loghin, “Optimization of High-order Non-uniform QAM Constellations," Proc. IEEE BMSB 2013, London, UK.

[19] B. Mouhouche, D. Ansorregui, and A. Mourad, "High Order Non-Uniform Constellations for broadcasting UHDTV," Proc. IEEE WCNC 2014, Istambul, Turkey.

[20] D. Perez-Calderon, V. Baena-Lecuyer, A. C. Oria, P. Lopez, and J. G. Doblado, "Simplified Rotated Constellation Demapper for Second Generation Terrestrial Digital Video Broadcasting,” IEEE Transactions on Broadcasting, vol. 59, no. 1, pp. 160-167, March 2013.

[21] ETSI TS 102831 v1.2.1, "Digital Video Broadcasting (DVB); Implementation guidelines for a second generation broadcasting system (DVB-T2)” August 2012.

Lachlan Michael received B.Eng (Hons) and B.A. (Japanese) from the University of Queensland, Australia in 1994, M.Eng from Keio University, Japan in 1997 and Ph.D. degree in Electrical Engineering from Keio University in 2000. He joined Sony Computer Science Laboratories in 2000 and moved to Sony Corporation in 2002.

Dr. Michael has been actively involved in both broadcast receiver development and broadcast standardization, having participated in DVB-T2/T2-Lite, DVB-C2, DVB-NGH and DVB-S2x activities. In DVB-T2 he led the V\&V (Verification and Validation) group concluding three successful plug-fests. He has participated in ATSC 3.0 standardization from the beginning, notably as the Chairman of the Modulation and Coding Ad-Hoc Group and editor of the A/322 specification.

David Gómez-Barquero received the double M.Sc. degrees in telecommunications engineering from the Universitat Politecnica de Valencia (UPV), Spain, and the University of Gävle, Sweden, in 2004, the Ph.D. degree in telecommunications from the UPV in 2009; and he carried out a 2-year post-doc at the Fraunhofer Heinrich Hertz Institute,
Germany. He is a Senior Researcher (Ramon \& Cajal Fellow) with the Institute of Telecommunications and Multimedia Applications, UPV, where he leads a research group working on next generation broadcasting technologies. Previously, he hold visiting research appointments at Ericsson Eurolab, Germany, the Royal Institute of Technology, Sweden, the University of Turku, Finland, the Technical University of Braunschweig, Germany, the Sergio Arboleda University of Bogota, Colombia, and the New Jersey Institute of Technology, USA.

Dr. Gómez-Barquero has been since 2008 actively participating in the European digital television standardization forum DVB in different topics such as upper layer forward error correction, DVB-T2, T2-Lite, and DVB-NGH. In 2013, he joined the U.S. digital television standardization forum ATSC to work on ATSC 3.0, acting as Vice-Chairman of the Modulation and Coding Ad-Hoc Group. He is the Editor of the book entitled Next Generation Mobile Broadcasting (CRC Press, 2013). 